PROCEEDINGS OF THE

AMERICAN MATHEMATICAL SOCIETY

Volume 127, Number 9, Pages 2779-2782

S 0002-9939(99)05344-7

Article electronically published on May 4, 1999

\title{
A COMPOSITION FORMULA IN THE RANK TWO FREE GROUP
}

\author{
FRANCISCO GONZÁLEZ-ACUÑA AND ARTURO RAMÍREZ
}

(Communicated by Ronald A. Fintushel)

\begin{abstract}
Using the fundamental group of a punctured torus, a free group $F$ of rank two, and the fact that the natural eipmorphism from AutF onto $\operatorname{Aut}\left(F / F^{\prime}\right)$ has as kernel the group of inner automorphisms of $F$, we describe representatives of the conjugacy classes of generating pairs of $F$ and give explicit relations between them.
\end{abstract}

Let $F=F(S, T)$ be the free group on $S$ and $T$. By a theorem of Nielsen [N] (see $\left[\mathrm{LS}\right.$, p. 25]) the natural epimorphism from $\operatorname{AutF}$ onto $\operatorname{Aut}\left(F / F^{\prime}\right)(=G L(2, \mathbb{Z}))$ has as kernel the group of inner automorphisms of $F$. From this it follows easily that, if $\alpha$ is the abelianization homomorphism from $F$ onto $F / F^{\prime}\left(=\mathbb{Z}^{2}\right)$ and $\mathbf{a} \in \mathbb{Z}^{2}$ is primitive $^{1}$, then the inverse image of a under $\alpha$ is a conjugacy class of primitive elements. Also, if $\left(\mathbf{a}_{1}, \mathbf{a}_{2}\right)$ is a basis of $\mathbb{Z}^{2}$, then, up to conjugacy, there is a unique basis $\left(f_{1}, f_{2}\right)$ of $F$ such that $\left(f_{i}\right) \alpha=\mathbf{a}_{i}(i=1,2)$. (The basis $\left(f_{1}, f_{2}\right)$ is conjugate to $\left(g_{1}, g_{2}\right)$ if there exists $w \in F$ such that $\left.w^{-1} f_{i} w=g_{i}(i=1,2)\right)$.

In the important paper [OZ], Osborne and Zieschang define explicitly primitive words $W_{m, n} \in F(S, T)$, where $m$ and $n$ are relatively prime integers, such that $\left(W_{m, n}\right) \alpha=(m, n)$. They also state that if $m n-p q=1$, then $\left(W_{m, n}, W_{p, q}\right)$ is a basis of $F$; this, while correct for nonnegative values of $m, n, p, q$, is not valid in general (for example $W_{-2,-3}$ and $W_{1,1}$ do not generate $F$ ). A composition formula is also stated in [OZ, Thm. 3.5] but this, even with the correction of indices in [LTZ, 2.1.3], is incorrect in general.

In the present article we consider elements $V_{\mathbf{a}}^{\varepsilon}$ of $F$ for $\mathbf{a}=(m, n) \in \mathbb{Z}^{2}$ and $\varepsilon \in \mathcal{D} \subset \mathbb{R}^{2}$ where $\mathcal{D}$ is the complement of the union of all the lines that intersect $\mathbb{Z}^{2}$ in more than one point. If $\operatorname{gcd}(m, n)=1$, then $V_{(m, n)}^{\varepsilon}$ is conjugate to $W_{m, n}$. We show in Theorem 1.i) that $\left(V_{\mathbf{a}}^{\varepsilon}, V_{\mathbf{b}}^{\varepsilon}\right)$ is a basis of $F$, if $\mathbb{Z}^{2}=\langle\mathbf{a}, \mathbf{b}\rangle$, and obtain in Theorem 1.ii) a composition formula. Everything is obtained by applying the fundamental group functor $\pi$ to the punctured torus.

Denote by $\mathbb{T}$ the torus $\mathbb{R}^{2} / \mathbb{Z}^{2}$, by $\mathbb{T}_{0}$ the punctured torus $\left(\mathbb{R}^{2}-\mathbb{Z}^{2}\right) / \mathbb{Z}^{2}$ and by $\rho: \mathbb{R}^{2}-\mathbb{Z}^{2} \rightarrow \mathbb{T}_{0}$ the natural projection. If $\mathbf{a} \in \mathbb{Z}^{2}$ and $\varepsilon \in \mathcal{D}$, then denote $(\varepsilon) \rho$ by $\bar{\varepsilon}$ and define $\gamma_{\mathbf{a}}^{\varepsilon} \in \pi\left(\mathbb{T}_{0}, \bar{\varepsilon}\right)$ as the homotopy class of the loop $(\varepsilon+t \mathbf{a}) \rho$, $t \in[0,1]$. Denote $\gamma_{(1,0)}^{\varepsilon}\left(\right.$ resp. $\left.\gamma_{(0,1)}^{\varepsilon}\right)$ by $S_{\varepsilon}\left(\right.$ resp. $\left.T_{\varepsilon}\right)$. There is an isomorphism

Received by the editors September 15, 1997.

1991 Mathematics Subject Classification. Primary 57M07, $20 \mathrm{E} 05$.

Key words and phrases. Rank two free group, primitive words.

${ }^{1}$ An element $f$ of a rank two group $G$ is primitive if there exists $g \in G$ such that $f$ and $g$ generate $G$. 
$\varphi_{\varepsilon}: F(S, T) \rightarrow \pi\left(\mathbb{T}_{0}, \bar{\varepsilon}\right)$ sending $S$ to $S_{\varepsilon}$ and $T$ to $T_{\varepsilon}$. Define $V_{\mathbf{a}}^{\varepsilon} \in F(S, T)$ by $\left(V_{\mathbf{a}}^{\varepsilon}\right) \varphi_{\varepsilon}=\gamma_{\mathbf{a}}^{\varepsilon}$. Notice that $\left(V_{\mathbf{a}}^{\varepsilon}\right) \alpha=\mathbf{a}$ since the inclusion induced homomorphism $\pi\left(\mathbb{T}_{0}, \bar{\varepsilon}\right) \rightarrow \pi(\mathbb{T}, \bar{\varepsilon})$ is the abelianization.

In comparison with $[\mathrm{OZ}, 4.2] V_{\mathbf{a}}^{\varepsilon}$ is represented by the word obtained by traveling along the segment, in $\mathbb{R}^{2}-\mathbb{Z}^{2}$, from $\varepsilon$ to $\varepsilon+\mathbf{a}$ and writing $S$ (resp. $S^{-1}$ ) whenever we cross a component of $\mathbb{Z} \times \mathbb{R}$ from left to right (resp. from right to left) and writing $T$ (resp. $T^{-1}$ ) whenever we cross a component of $\mathbb{R} \times \mathbb{Z}$ from below (resp. from above).

A matrix $M=\left(\begin{array}{l}\mathbf{a} \\ \mathbf{b}\end{array}\right) \in G L(2, \mathbb{Z})$ defines a linear automorphism of $\left(\mathbb{R}^{2}, \mathbb{Z}^{2}\right)$ that, for any $\varepsilon \in \mathcal{D}$, induces a homeomorphism $\mu_{M}^{\varepsilon}:\left(\mathbb{T}_{0}, \bar{\varepsilon}\right) \rightarrow\left(\mathbb{T}_{0}, \overline{\varepsilon M}\right)$. This induces $\nu_{M}^{\varepsilon}$ : $\pi\left(\mathbb{T}_{0}, \bar{\varepsilon}\right) \stackrel{\sim}{\rightarrow} \pi\left(\mathbb{T}_{0}, \overline{\varepsilon M}\right)$ and an automorphism $\Psi_{M}^{\varepsilon}$ of $F$ defined by $\Psi_{M}^{\varepsilon}=\varphi_{\varepsilon} \nu_{M}^{\varepsilon} \varphi_{\varepsilon M}^{-1}$. We have $\gamma_{\mathbf{c}}^{\varepsilon} \nu_{M}^{\varepsilon}=\gamma_{\mathbf{c} M}^{\varepsilon M}$ since $\rho \mu_{M}^{\varepsilon}=M \rho$ and therefore $V_{\mathbf{c}}^{\varepsilon} \Psi_{M}^{\varepsilon}=V_{\mathbf{c} M}^{\varepsilon M}$ for all $\mathbf{c} \in \mathbb{Z}^{2}$ (this equality was suggested by the referee). In particular $(S) \Psi_{M}^{\varepsilon}=V_{\mathbf{a}}^{\varepsilon M}$ and $(T) \Psi_{M}^{\varepsilon}=V_{\mathbf{b}}^{\varepsilon M}$. Notice that, for any word $W(S, T)$ we have $(W(S, T)) \Psi_{M}^{\varepsilon}=$ $W\left((S) \Psi_{M}^{\varepsilon},(T) \Psi_{M}^{\varepsilon}\right)=W\left(V_{\mathbf{a}}^{\varepsilon M}, V_{\mathbf{b}}^{\varepsilon M}\right)$. One has the following composition theorem.

Theorem 1. We have: i) If $\langle\mathbf{a}, \mathbf{b}\rangle=\mathbb{Z}^{2}$ and $\varepsilon \in \mathcal{D}$, then $\left\langle V_{\mathbf{a}}^{\varepsilon}, V_{\mathbf{b}}^{\varepsilon}\right\rangle=F$.

ii) If $M, N \in G L(2, \mathbb{Z})$ and $\varepsilon \in \mathcal{D}$, then $\Psi_{N M}^{\varepsilon}=\Psi_{N}^{\varepsilon} \Psi_{M}^{\varepsilon N}$.

iii) If $\mathbf{c} \in \mathbb{Z}^{2}$, then $V_{\mathbf{c} M}^{\varepsilon}=V_{\mathbf{c}}^{\varepsilon M^{-1}}\left(V_{\mathbf{a}}^{\varepsilon}, V_{\mathbf{b}}^{\varepsilon}\right)$ where $M=\left(\begin{array}{l}\mathbf{a} \\ \mathbf{b}\end{array}\right) \in G L(2, \mathbb{Z})$.

Proof. Since $(S) \Psi_{M}^{\varepsilon M^{-1}}=V_{\mathbf{a}}^{\varepsilon}$ and $(T) \Psi_{M}^{\varepsilon M^{-1}}=V_{\mathbf{b}}^{\varepsilon}$, i) follows.

As $\mu_{N M}^{\varepsilon}=\mu_{N}^{\varepsilon} \mu_{M}^{\varepsilon N}$ we obtain $\nu_{N M}^{\varepsilon}=\nu_{N}^{\varepsilon} \nu_{M}^{\varepsilon N}$; therefore $\Psi_{N M}^{\varepsilon}=\varphi_{\varepsilon} \nu_{N M}^{\varepsilon} \varphi_{\varepsilon N M}^{-1}=$ $\varphi_{\varepsilon} \nu_{N}^{\varepsilon} \nu_{M}^{\varepsilon N} \varphi_{\varepsilon N M}^{-1}=\varphi_{\varepsilon} \nu_{N}^{\varepsilon} \varphi_{\varepsilon N}^{-1} \varphi_{\varepsilon N} \nu_{M}^{\varepsilon N} \varphi_{\varepsilon N M}^{-1}=\Psi_{N}^{\varepsilon} \Psi_{M}^{\varepsilon N}$ which proves ii).

The identities $(W(S, T)) \Psi_{M}^{\varepsilon}=W\left(V_{\mathbf{a}}^{\varepsilon M}, V_{\mathbf{b}}^{\varepsilon M}\right)$ and $V_{\mathbf{c}}^{\varepsilon} \Psi_{M}^{\varepsilon}=V_{\mathbf{c} M}^{\varepsilon M}$ give $V_{\mathbf{c} M}^{\varepsilon M}=$ $V_{\mathbf{c}}^{\varepsilon}\left(V_{\mathbf{a}}^{\varepsilon M}, V_{\mathbf{b}}^{\varepsilon M}\right)$ that is equivalent to iii).

Remarks. 1. By varying $\varepsilon^{\prime}$ along the segment from $\varepsilon$ to $\varepsilon+\mathbf{a}$ we obtain as $V_{(m, n)}^{\varepsilon^{\prime}}$ all the cyclic permutations of $V_{(m, n)}^{\varepsilon}$; also if $\varepsilon^{\prime}, \varepsilon \in \mathcal{D}$, then $V_{(m, n)}^{\varepsilon^{\prime}}$ is a cyclic permutation of $V_{(m, n)}^{\varepsilon}$ since they are conjugate and cyclically reduced. Hence, if $\operatorname{gcd}(m, n)=1$, then $\left\{V_{(m, n)}^{\varepsilon}: \varepsilon \in \mathcal{D}\right\}$ is the set of the primitive elements of $F$ whose image under $\alpha$ is $(m, n)$ and have minimal length (equal to $|m|+|n|$ ); this set, which contains $W_{m, n}$, has cardinality $|m|+|n|$ since $V_{(m, n)}^{\varepsilon}$ is not a proper power (cf. [OZ, 4.2]).

2. In Theorem 1.ii) one needs different superscripts: There is no collection $\left\{\Psi_{M} \in A u t F: M \in G L(2, \mathbb{Z})\right\}$ such that the image of any $\Psi_{M}$ under $\lambda: A u t F \rightarrow$ OutF is $M$ and $\Psi_{N M}=\Psi_{N} \Psi_{M}$. That is, $\lambda$ does not split since there are elements of order 6 in OutF but not in AutF (see [LS, I.4.6]).

However there is a collection $\left\{\Psi_{M} \in A u t F: M \in G L^{+}(2, \mathbb{Z})\right\}$, where $G L^{+}(2, \mathbf{Z})$ $=\left\{\left(\begin{array}{cc}m & n \\ p & q\end{array}\right) \in G L(2, Z): m \geq 0, n \geq 0, p \geq 0, q \geq 0\right\}$, such that $\lambda\left(\Psi_{M}\right)=M$ and $\Psi_{N M}=\Psi_{N} \Psi_{M}$. To see this observe that $S L^{+}(2, \mathbb{Z})$, the set of matrices in $S L(2, \mathbb{Z})$ with nonnegative entries, is a free monoid on $A$ and $B A B$ where $A=\left(\begin{array}{cc}1 & 1 \\ 0 & 1\end{array}\right), B=$ $\left(\begin{array}{ll}0 & 1 \\ 1 & 0\end{array}\right)$ [CMZ, Lemma 3.5] and from this one obtains the monoid presentation $(A, B$ : $\left.B^{2}\right)$ of $G L^{+}(2, \mathbf{Z})$, that is, every element of $G L^{+}(2, \mathbf{Z})$ can be written uniquely as a word in $A$ and $B$ where the exponents of $A$ are positive and the exponents of $B$ are 1. One can define $(S) \Psi_{A}=S T,(T) \Psi_{A}=T,(S) \Psi_{B}=T,(T) \Psi_{B}=S$ and if $M=B^{\delta_{1}}\left(\prod_{i=1}^{n} A^{e_{i}} B\right) B^{\delta_{2}}, n>0, e_{i}>0(i=1, \ldots, n), \delta_{j}=0,1(j=1,2)$, we define $\Psi_{M}=\Psi_{B}^{\delta_{1}}\left(\prod_{i=1}^{n} \Psi_{A}^{e_{i}} \Psi_{B}\right) \Psi_{B}^{\delta_{2}}$. The $\Psi$ 's have the desired properties. 
3. By Theorem 1.iii) if $M=\left(\begin{array}{l}\mathbf{a} \\ \mathbf{b}\end{array}\right)$ and $\left(\eta_{1}, \eta_{2}\right)=\varepsilon M^{-1}$, then we have a general addition formula that implies Theorem 1.3 of $[\mathrm{OZ}]$ :

$$
V_{\mathbf{a}+\mathbf{b}}^{\varepsilon}=V_{(1,1)}^{\left(\eta_{1}, \eta_{2}\right)}\left(V_{\mathbf{a}}^{\varepsilon}, V_{\mathbf{b}}^{\varepsilon}\right)= \begin{cases}V_{\mathbf{a}}^{\varepsilon} V_{\mathbf{b}}^{\varepsilon} \text { if } \eta_{1}-\left[\eta_{1}\right]>\eta_{2}-\left[\eta_{2}\right], \\ V_{\mathbf{b}}^{\varepsilon} V_{\mathbf{a}}^{\varepsilon} \text { if } \eta_{1}-\left[\eta_{1}\right]<\eta_{2}-\left[\eta_{2}\right] .\end{cases}
$$

4. It may be desirable to modify slightly the definition of the words $W_{m, n}$ given in [OZ] as follows: If $n \geq 0$ define the $W_{m, n}$ as in [OZ], but if $n<0$ define $W_{m, n}$ as $W_{-m,-n}^{-1}$ not $W_{m,-n}\left(S, T^{-1}\right)$, as stated in [OZ]. With this modification the analog of Theorem 1.i) holds, that is, if $m q-n p= \pm 1$, then $\left\langle W_{m, n}, W_{p, q}\right\rangle=F$.

5 . If we let $\varepsilon$ be a pair of infinitesimals [SL] that does not lie in a line in $* \mathbb{R}^{2}$ intersecting $\mathbb{Z}^{2}$ in more than one point and if $\mathbf{a} \in \mathbb{Z}^{2}$, then $V_{\mathbf{a}}^{\varepsilon}$ can be defined as in the fifth paragraph. Again $\left\langle V_{\mathbf{a}}^{\varepsilon}, V_{\mathbf{b}}^{\varepsilon}\right\rangle=F$ if $\langle\mathbf{a}, \mathbf{b}\rangle=\mathbb{Z}^{2}$ and the assertion 1.iii) is still valid. Then, with our modification of the definition of $W_{m, n}$ given in the previous remark, $W_{m, n}=V_{(m, n)}^{\left(-\delta i^{2},-i\right)}$ where $i$ is a positive infinitesimal and $\delta=\left\{\begin{array}{l}1 \text { if } m n \geq 0 \\ -1 \text { if } m n<0 .\end{array}\right.$

If $k l \neq 0$ and $\operatorname{gcd}(k, l)=1$, the axes and the line through the origin and $(k, l)$ divide the plane ${ }^{*} \mathbb{R}^{2}$ into six open regions. If the infinitesimal pairs $\varepsilon$ and $\varepsilon^{\prime}$ belong to the same region, then $V_{(k, l)}^{\varepsilon}=V_{(k, l)}^{\varepsilon^{\prime}}$. If $V_{k, l}^{\prime}$ is the word defined by the open segment from $(0,0)$ to $(k, l)$ (cf. [OZ, Definition 2.1]), $\delta_{1}=\operatorname{sgn} k$ and $\delta_{2}=$ $\operatorname{sgn} l$, then $V_{(k, l)}^{\varepsilon}$ is one of the words $S^{\delta_{1}} T^{\delta_{2}} V_{k, l}^{\prime}, T^{\delta_{2}} S^{\delta_{1}} V_{k, l}^{\prime}, S^{\delta_{1}} V_{k, l}^{\prime} T^{\delta_{2}}, T^{\delta_{2}} V_{k, l}^{\prime} S^{\delta_{1}}$, $V_{k, l}^{\prime} S^{\delta_{1}} T^{\delta_{2}}$ or $V_{k, l}^{\prime} T^{\delta_{2}} S^{\delta_{1}}$ depending on the region in which $\varepsilon$ lies.

Let $\vec{W}_{k, l}=V_{k, l}^{i(l+i,-k+\sqrt{2} i)}$ and $\overleftarrow{W}_{k, l}=V_{k, l}^{-i(l+i,-k+\sqrt{2} i)}$, thus, if $k>0, l>0$ and $\operatorname{gcd}(k, l)=1$, then $\vec{W}_{k, l}=T V_{k, l}^{\prime} S$ and $\overleftarrow{W}_{k, l}=S V_{k, l}^{\prime} T$. Now, if $k, l, n$ and $q$ are nonnegative integers, $m>0, p>0$ with $\operatorname{gcd}(k, l)=1$ and $m q-n p=d= \pm 1$, then

$$
W_{k m+l p, k n+l q}= \begin{cases}\vec{W}_{k, l}\left(W_{m, n}, W_{p, q}\right) & \text { if } d=1 \\ \overleftarrow{W}_{k, l}\left(W_{m, n}, W_{p, q}\right) & \text { if } d=-1\end{cases}
$$

This follows from Theorem 1.iii) taking $\varepsilon=\left(-i^{2},-i\right)$ and $M=\left(\begin{array}{cc}m & n \\ p & q\end{array}\right)$; then $V_{(k, l)}^{\varepsilon M^{-1}}=\left\{\begin{array}{ll}\vec{W}_{k, l} & \text { if } d=1, \\ \overleftarrow{W}_{k, l} & \text { if } d=-1 .\end{array}\right.$ This gives the modification needed in [OZ, Theorem 3.5] and [LTZ, 2.1.3].

We thank the referee for the suggestions, which improved the presentation of the paper.

\section{REFERENCES}

[CMZ] M. Cohen, W. Metzler and A. Zimmermann, What Does a Basis of F $(a, b)$ Look Like?, Math. Ann. 257 (1981), 435-445. MR 82m:20028

[LTZ] M. Lustig, E-M. Thiele and H. Zieschang, Primitive Elements in the Free Product of Two Finite Cyclic Groups, Abh. Math. Sem. Univ. Hamburg 65 (1995), 277-281. MR 96g:20035

[LS] R. Lyndon and P. Schupp, Combinatorial Group Theory, Springer-Verlag 1977. MR $\mathbf{5 8 : 2 8 1 8 2}$

[N] J. Nielsen, Die Isomorphismen der allgemeinen, unendlichen Gruppe mit zwei Erzeugenden, Math. Ann. 78 (1918), 385-397. 
[OZ] R. Osborne and H. Zieschang, Primitives in the Free Group on Two Generators, Invent. Math. 63 (1981), 17-24. MR 82i:20042

[SL] K. Stroyan and W. Luxemburg, Introduction to the theory of infinitesimals, Academic Press 1976. MR 58:10429

Instituto de Matemáticas, UnAM, México D.F. 04510, Mexico; Cimat, Guanjuato AP 402 Guanajuato 36000, MÉxico

E-mail address: fico@matem.unam.mx

Cimat, Guanjuato AP 402 Guanajuato 36000, México

E-mail address: ramirez@fractal.cimat.mx 